\title{
DIVERSIDAD DE LOS PECES DULCEACUÍCOLAS EN RESBALADERO, HOLGUÍN, CUBA
}

\author{
Diversity of freshwater fishes in Resbaladero, Holguin, Cuba
}

\author{
Enrique Reynaldo de la Cruz ${ }^{\mathrm{a}^{*}}$, David F. Hernández Marrero ${ }^{\mathrm{b}}$, \\ Jacobo Urbino Rodríguez ${ }^{\mathrm{c}}$ y Roberto Ayra Cedeño ${ }^{\mathrm{d}}$
}

Centro de Investigaciones y Servicios Ambientales de Holguín. Calle 18 e/1ra y Maceo, Rpto. El Llano. Apartado Postal 80100. Holguín, Cuba. a (D) orcid.org/0000-0003-3790-9843; b (1) orcid.org/0000-0001-6963-3875, david@cisat.cu; c (1) orcid.org/0000-0001-6519-8394, jacobo@cisat.cu; d(i) orcid.org/0000-0002-8619-797X, rayrac49@gmail.com.*Para correspondencia: ereynaldodelacruz@gmail.com

[Recibido: 21 de julio, 2021. Aprobado para publicación: 10 de noviembre, 2021]

\section{RESUMEN}

El objetivo de este estudio fue determinar la diversidad de los peces dulceacuícolas en diferentes épocas del año en Resbaladero, Holguín, Cuba. Nueve especies fueron registradas en ambos periodos del año, ubicados en tres órdenes, seis familias y ocho géneros. Las especies que presentaron las mayores densidades en ambas épocas del año fueron Girardinus denticulatus $\left(9.85\right.$ y $\left.5.34 \mathrm{ind} / \mathrm{m}^{2}\right)$ y Gambusia punticulata $\left(9.36\right.$ y $\left.6.17 \mathrm{ind} / \mathrm{m}^{2}\right)$, densidades correspondientes a las épocas de seca y lluvia. El sitio con la mayor riqueza de especies en ambos periodos fue presa de Colorado con siete especies en seca y seis en lluvia. A diferencia de pozo Urbano Pérez con una sola especie en ambos periodos del año. En relación a la equitatividad en ambas épocas, los sitios con los mayores valores fueron arroyo Claro Bajo 2 y arroyo Claro Bajo. La dominancia ecológica fue mayor en ambas épocas del año en pozo Urbanos Pérez. Presa Colorado fue el sitio de mayor diversidad en ambos periodos, con valores de 1.17 y 1.44, respectivamente. Presa Colorado fue el lugar que presentó menos intercambio de especies con el resto de los sitios, siendo significativo en la época de seca con pozo Los Ninos (1.0) y época de lluvia con arroyo Claro Bajo (0.75). Se estableció que las comunidades ictiológicas en Resbaladero están estructuradas por el remplazamiento entre especies. El análisis de similitud permitió conformar tres grandes grupos en época de seca y dos en lluvia. Las especies que más contribuyeron en la disimilitud fueron Girardinus dintuculatus, G. punticulata y G. punctata.

Palabras clave: ecosistemas, índices ecológicos, periodos del año.

\section{ABSTRACT}

The objective of this study was to determine the diversity of freshwater fish at different times of the year in Resbaladero, Holguin, Cuba. Nine species were recorded in both periods of the year, located in three orders, six families and eight genera. The species with the highest densities at both times of the year are Girardinus denticulatus $\left(9.85\right.$ and $\left.5.34 \mathrm{ind} / \mathrm{m}^{2}\right)$ and Gambusia punticulata $\left(9.36\right.$ and $\left.6.17 \mathrm{ind} / \mathrm{m}^{2}\right)$, densities corresponding to the dry and rainy season. 
The site with the highest species richness in both periods was Colorado dam with seven species in dry and six in rainy. Unlike the Urbano Pérez water well with only one species in both periods of the year. In relation to fairness in both periods, the sites with the highest values were Claro Bajo 2 stream and Claro Bajo stream. Colorado dawn was the site with the greatest diversity in both periods, with values of 1.17 and 1.44, respectively. Colorado dam was the place that presented the least number of replacements among species in the dry season with the Los Ninos (1.0) water well and in the rainy season with the Claro Bajo stream (0.75). The gamma diversity allowed to establish that the replacement between species predominates in this set of communities. The similarity analysis made it possible to form three large groups in the dry season and two in the rainy season. The species that contributed the most to the dissimilarity were G. denticulatus, G. punticulata and G. punctata.

Keywords: ecosystems, ecological indices, periods of the year.

\section{INTRODUCCIÓN}

En Cuba se han identificado 57 especies de peces dulceacuícolas. De ellas, 30 se clasifican como estrictas de agua dulce y 23 endémicas (Ponce de León et al., 2014). Para la provincia de Holguín se reportan 25 especies, comprendidas en la clase Actinopterygii, ubicadas en ocho órdenes, 13 familias y 20 géneros, para un $36 \%$ de endemismo; 11 estrictamente de agua dulce. La familia Poeciliidae es la de mayor riqueza. Los géneros mejor representados son Gambusia, Girardinus y Nandopsis (Vega et al., 2017).

Las principal problemática que atenta contra la conservación de las especies ícticas dulceacuícolas en Cuba es la acción sinérgica de múltiples estresores: contaminación, represamiento, reducción de nutrientes, degradación de los hábitats, cambio climático, sobrepesca, etc. Estos factores provocan la disminución de los peces en Cuba. Representando un peligro mayor la introducción de especies de peces exóticos en reservorios naturales, ocasionando en la fauna autóctona la extinción y deterioro de su hábitat (Quammen, 1996), constituyendo la principal problemática en Cuba cuyo efecto no está bien documentado aún (Ponce de León et al., 2014; Reynaldo et al., 2016, 2021; Vega et al., 2017). Además del desconocimiento generalizado de su historia de vida (Ponce de León y Rodríguez, 2010).

En la provincia de Holguín, las especies exóticas con mayor distribución son Poecilia reticulata, Oreochromis aureus, Ctenopharyngodon idella y Clarias gariepinus (Reynaldo et al., 2016, 2021; Vega et al., 2017). En Cuba, se desconoce en su mayoría el estado de conservación de las poblaciones de los peces dulceacuícolas (Ponce de León y Rodríguez, 2010). La contribución al conocimiento de la ictiofauna dulceacuícola de Resbaladero se encuentra limitada a observaciones puntuales. Además de la diversidad ictiológica y su variabilidad en diferentes épocas del año.

\section{OBJETIVOS}

- Determinar la diversidad y similitud de los peces dulceacuícolas en diferentes épocas del año en Resbaladero, Holguín, Cuba. 


\section{MATERIALES Y MÉTODOS}

El área de estudio se enmarca desde Presa Colorado (N 20 59' 45.4”; W 076 08' 07.0”) hasta arroyo Claro Bajo 2 (N 20 59' 31.8"; W 076 10' 20.5"), (Fig. 1). Los muestreos se realizaron en dos épocas del año 2019 (seca y lluvia). El área de trabajo comprendió casi toda la totalidad de Resbaladero, localizado en el municipio de Rafael Freyre, en la provincia de Holguín. Se seleccionaron seis sitios de muestreo (Fig. 1; Tabla I). Cada estación fue georeferenciada con Sistema de Posicionamiento Global, GPS (Garmin 12XL). Los organismos capturados se preservaron en pomos con etanol al $90 \%$ y en formol al $10 \%$, a $4{ }^{\circ} \mathrm{C}$, identificados posteriormente según: Ponce de León y Rodríguez (2010) y Vega et al. (2017).

Diseño de muestreo. Las estaciones fueron seleccionadas siguiendo las dimensiones de sus caudales y la confluencia de los mismos. Se realizó un muestreo puntual, dentro de cada reservorio de agua, empleando un jamo de $3 \mathrm{~mm}$ de luz de malla (45 x 50 x $180 \mathrm{~mm}$ ) para los peces de menor tamaño y una red de $2 \mathrm{~m}$ de longitud y $1 \mathrm{~m}$ de cuerpo con lastre inferior para los peces de mayor talla en los meses de febrero y septiembre del 2019. Para homogenizar la fuerza de muestreo se hizo 10 lances con cada arte de pesca.

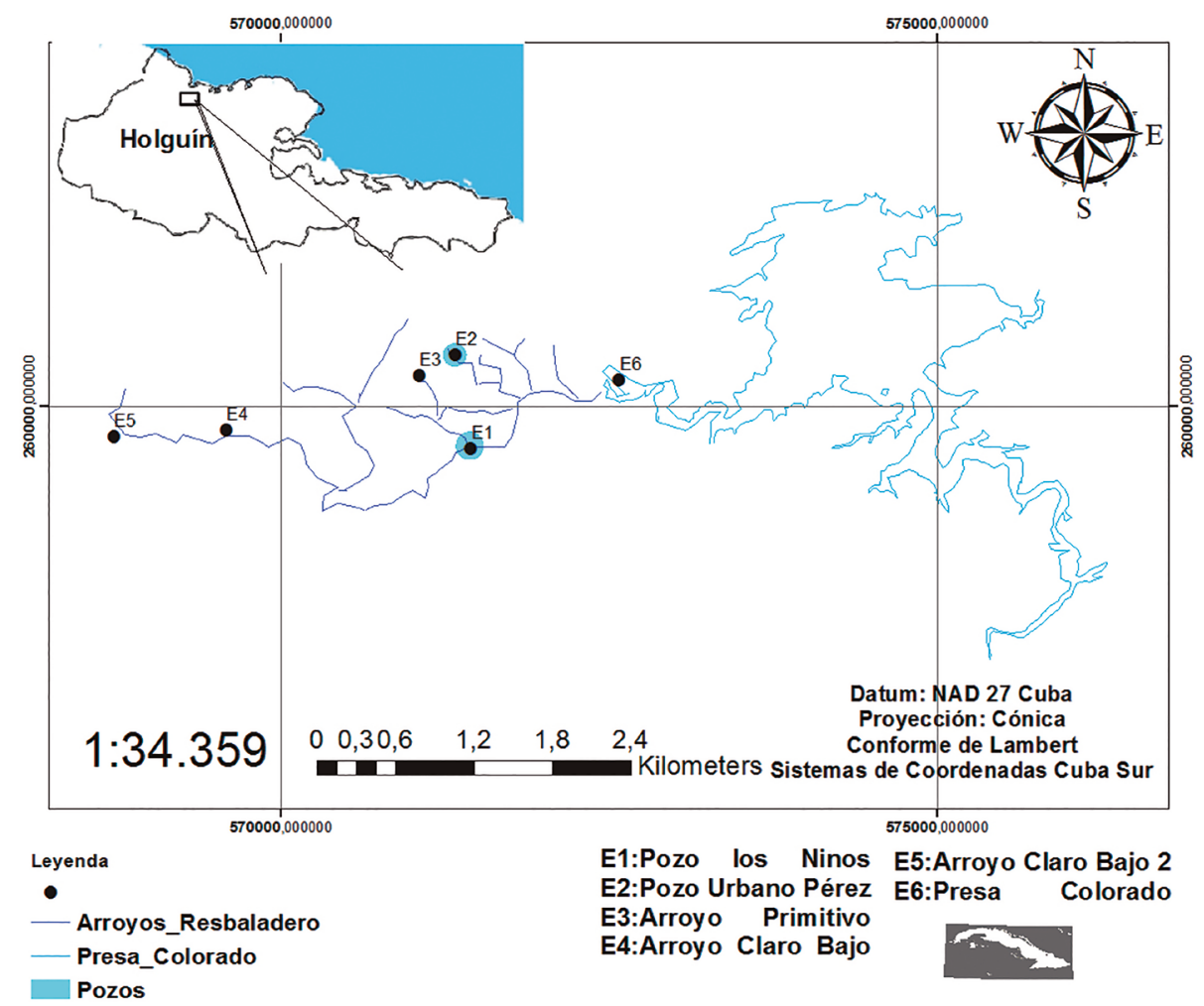

Figura 1. Ubicación geográfica de las comunidades ictiológicas en Resbaladero, Holguín, Cuba. 
Tabla I. Localidades de muestreos y coordenadas geográficas

\begin{tabular}{lccc}
\hline Localidades & Estaciones & Latitud (gg mm ss) & Longitud (gg mm ss) \\
\hline Pozo los Ninos & E1 & $20^{\circ} 53^{\prime} 21.6^{\prime \prime}$ & $076^{\circ} 16^{\prime} 31.4^{\prime \prime}$ \\
Pozo Urbano Pérez & E2 & $20^{\circ} 53^{\prime} 31.6^{\prime \prime}$ & $076^{\circ} 10^{\prime} 39.4^{\prime \prime}$ \\
Arroyo Primitivo & E3 & $20^{\circ} 59^{\prime} 46.7^{\prime \prime}$ & $076^{\circ} 08^{\prime} 59.7^{\prime \prime}$ \\
Arroyo Claro Bajo & E4 & $20^{\circ} 59^{\prime} 33.3^{\prime \prime}$ & $076^{\circ} 09^{\prime} 50.8^{\prime \prime}$ \\
Arroyo Claro Bajo 2 & E5 & $20^{\circ} 59^{\prime} 31.8^{\prime \prime}$ & $076^{\circ} 10^{\prime} 20.5^{\prime \prime}$ \\
Presa Colorado & E6 & $20^{\circ} 59^{\prime} 45.4^{\prime \prime}$ & $076^{\circ} 08^{\prime} 07.0^{\prime \prime}$ \\
\hline
\end{tabular}

Análisis de los datos. Se determinó la abundancia numérica de cada especie (ind. $/ \mathrm{m}^{2}$ ), para el análisis de la estructura de las comunidades ictiológicas, contando número de individuos capturados por especies para un transecto de $1 \mathrm{~m}^{2}$. Se estimó la riqueza de especies ictiológicas (S) se estableció contando el número de especies por sitio. La equitatividad se estimó por Equidad de Pielou, índice que mide la proporción de la diversidad observada con relación a la máxima diversidad esperada. Su valor va de 0 a 1 , de forma que 1.0 corresponde a situaciones donde todas las especies son igualmente abundantes (Magurran, 1988). La dominancia de las especies se determinó por el índice de dominancia-D (Magurran, 1988). Se estableció la diversidad a nivel de comunidad con el índice de diversidad de Shannon (Moreno, 2001).

La diversidad beta fue determinada por el índice de Whittaker, (1972) este índice establece la diversidad entre hábitats y el grado de reemplazamiento de especies o cambio biótico a través de gradientes ambientales. La diversidad Gamma se obtuvo siguiendo los criterios, Diversidad gamma $=$ alfa promedio + beta, a partir de la riqueza de especies (Landen, 1996):

$$
\text { Beta }=\sum_{\mathrm{j}} \mathrm{qj}(\mathrm{ST}-\mathrm{Sj})
$$

donde:

Beta $=$ Diversidad Beta

$\mathrm{q} j=$ peso proporcional de la comunidad $j$, basado en su área o cualquier otra medida de importancia relativa.

$\mathrm{ST}=$ Número total de especies registradas en el conjunto de comunidades.

$\mathrm{Sj}=$ Número de especies registradas en la comunidad $j$.

Se comparó los índices ecológicos, a partir de la prueba de permutación de diversidad con 999 remplazamientos (Harper, 1999). En todos los análisis de diversidad se realizó el procesamiento de Bootstrap $\mathrm{N}=10$ 000, tipo centrado, intervalo de confianza del $95 \%$.

Se empleó un análisis de agrupamiento para representar las asociaciones de peces utilizando como medida de similitud el índice de Bray-Curtis. Este análisis constituye uno de los modelos más utilizados para cuantificar la semejanza entre comunidades a partir de datos cuantitativos. 
Para determinar las diferencias estadísticas entre los agrupamientos (40\% de similitud), se realizó un análisis de similaridades (ANOSIM) empleando 10000 permutaciones y una prueba SIMPER para establecer la contribución de la abundancia de las especies que más influyeron en la disimilitud entre los grupos (Clarke, 1993). El programa utilizado fue Past. 4.06b (Hammer et al., 2001).

\section{RESULTADOS}

Densidades en diferentes épocas del año. Se determinó un total de nueve especies en ambos periodos del año, ubicados en tres órdenes, seis familias y ocho géneros. Del total de especies, tres son endémicas y autóctonas respectivamente y el resto constituye especies introducidas. Las especies que presentaron las mayores densidades en ambas épocas del año en el área de estudio fueron Girardinus denticulatus (9.85 ind $/ \mathrm{m}^{2}$, lluvia) y (5.34 ind $/ \mathrm{m}^{2}$, seca) y Gambusia punticulata, 9.36 y $6.17 \mathrm{ind} / \mathrm{m}^{2}$ en ambos periodos del año respectivamente. La especie con mayores densidades en el periodo de seca en relación con la época de lluvia es G. punctata, especie endémica de Cuba, 2.47 y $1.34 \mathrm{ind} / \mathrm{m}^{2}$ respectivamente. Ctenopharyngodon idella, pez invasor, fue la especie con menor densidad, $0.3 \mathrm{ind} / \mathrm{m}^{2}$ en época de seca, no reportándose en la de lluvia, al igual que $O$. aureus con densidad de $\left(0.83\right.$ y $\left.1.24 \mathrm{ind} / \mathrm{m}^{2}\right)$ en ambos periodos del año (Fig. 2).

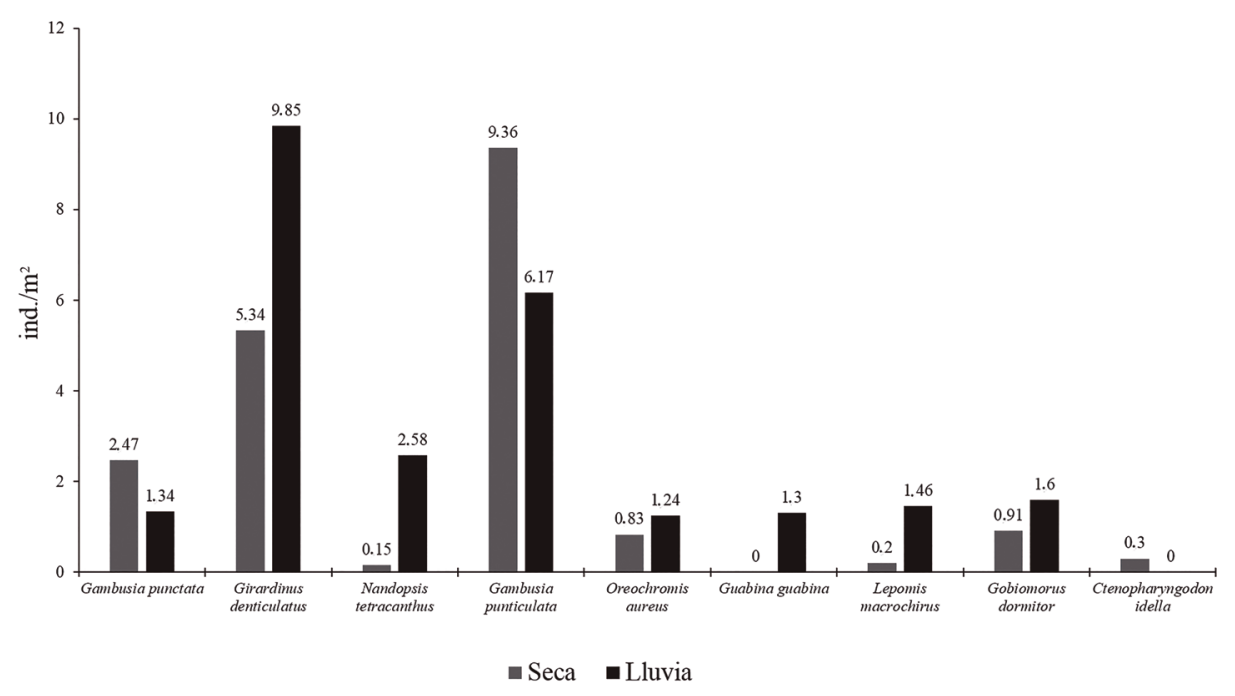

Figura 2. Densidad de las especies en diferentes épocas del año.

Análisis de los índices ecológicos. El sitio con la mayor riqueza de especies en ambos periodos del año fue presa de Colorado, con siete especies en el periodo de seca y seis en lluvia; a diferencia de pozo Urbano Pérez, con una sola especie en ambos periodos del año: G. denticulatus con densidades en época de seca $4.30 \mathrm{ind} / \mathrm{m}^{2}$ y lluvia $9.85 \mathrm{ind} / \mathrm{m}^{2}$ respectivamente. Al igual que pozo los Ninos y arroyo Primitivo, que presentaron una sola especie en el periodo de seca: G. punctata $\left(2.24 \mathrm{ind} / \mathrm{m}^{2}\right)$ y $G$. denticulatus $\left(1.04 \mathrm{ind} / \mathrm{m}^{2}\right)$, respectivamente, ambas especies endémicas de Cuba. Destacando que las dos últimas especies mencionadas no fueron registradas en la época de lluvia en los sitios donde se reportaron en el periodo de seca. Para la riqueza de especies, se encontró diferencias estadísticas significativas durante el periodo de seca, entre pozo Los Ninos y arroyo Primitivo $(\mathrm{p}=0.0001)$ y en ambos periodos del año entre presa Colorado y pozo Urbano Pérez $(\mathrm{p}=0.0001)$, (Fig. 3). 


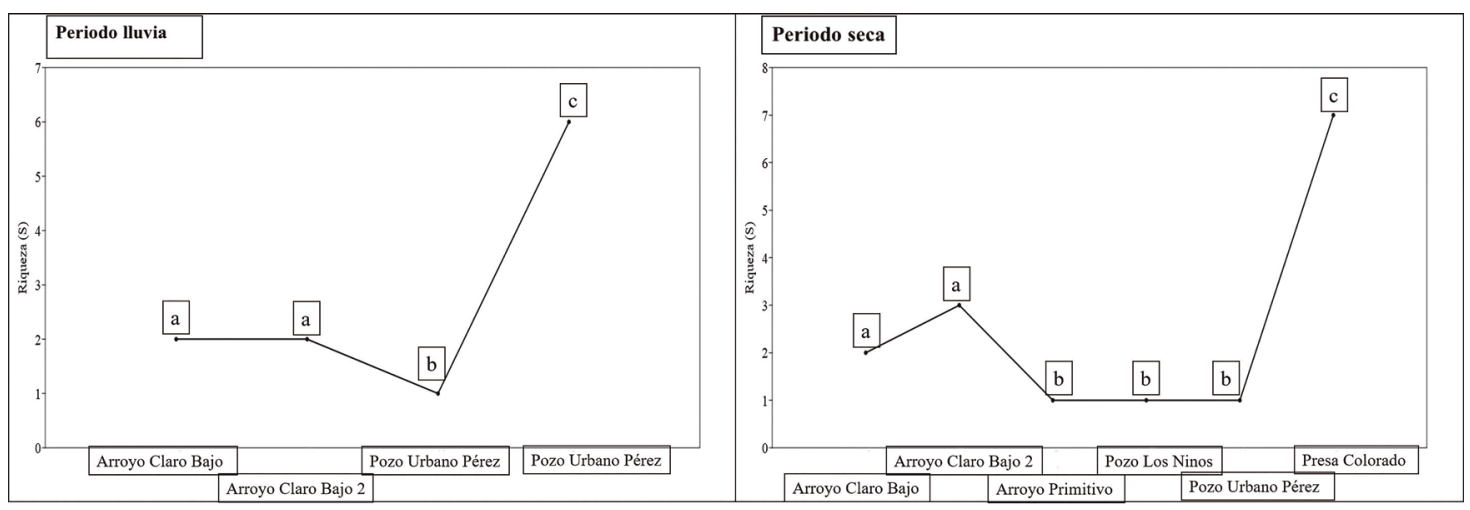

Figura 3. Riqueza de especies entre sitios en diferentes épocas del año. Letras diferentes entre grupos significan diferencias estadísticas a partir de la prueba Permutación de Diversidad para 999 remplazamientos.

En relación a la equitatividad para el período de seca, los sitios con los mayores valores fueron arroyo Claro Bajo 2 (0.69) y arroyo Claro Bajo (0.58). Las especies que influyeron en estos valores de igualdad ecológica en ambos sitios fueron $G$. punctata y G. denticulatus, además de Nandopsis tetracanthus presente en arroyo Claro Bajo 2, especies con abundancias similares. A diferencia de los pozos Urbanos Pérez, Los Ninos y arroyo Primitivo que no presentaron equitatividad. Para la época de lluvia, arroyo Claro bajo (0.93), arroyo Claro Bajo 2 (0.80) y presa Colorado (0.80), constituyen los sitios de mayor equitatividad ecológica. A diferencia de pozo Urbano Pérez que no presentó equidad (Fig. 4).

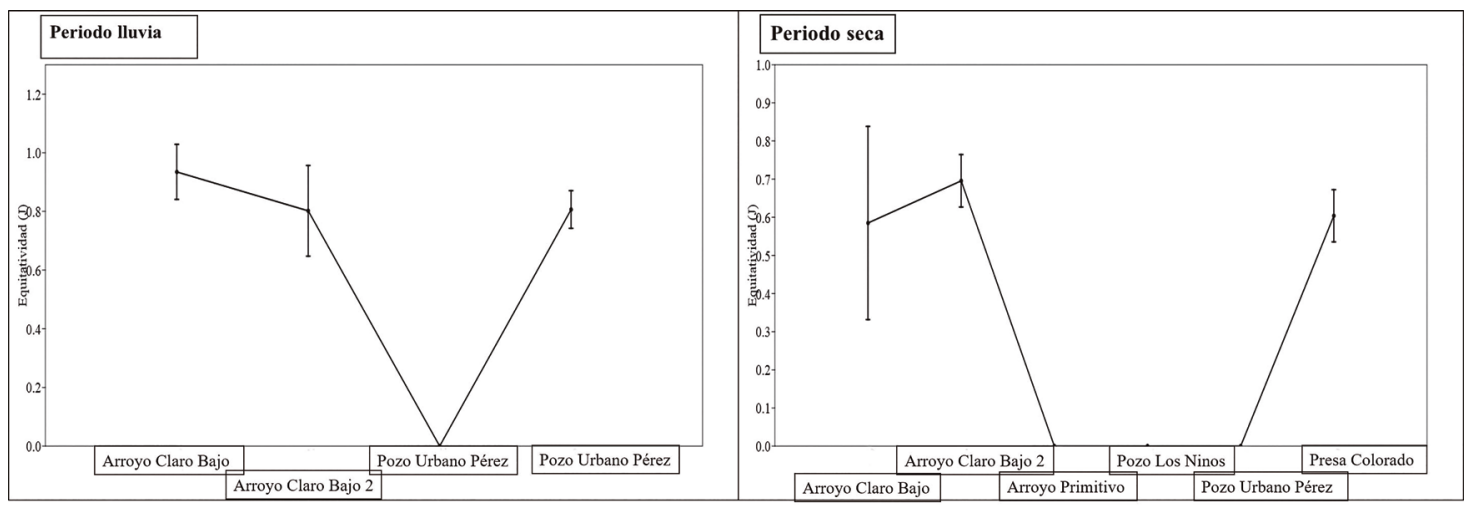

Figura 4. Equitatividad (J) de especies entre sitios en diferentes épocas del año.

La dominancia ecológica fue mayor en el periodo de seca en los sitios: arroyo Primitivo, pozos Los Ninos y Urbanos Pérez, este último en ambos periodos del año, cada sitio con valores de uno respectivamente. A diferencia de presa Colorado, que presentó los más bajos valores en ambas épocas del año, 0.4 y 0.28 respectivamente para seca y lluvia, al igual que arroyo Claro Bajo 2 en la época de poca lluvia (0.50). Las principales diferencias estadísticas fueron encontradas en el periodo de seca entre arroyo Claro Bajo 2 con arroyo Primitivo; pozo los Ninos y Urbano Pérez. Además de presa Colorado con los sitios de mayor abundancia $(\mathrm{p}=0.0001)$. En la época de lluvia destacó presa Colorado y arroyo Claro Bajo con pozo Urbano Pérez $(\mathrm{p}=0.0001)$ con las mayores diferencias estadísticas (Fig. 5). 


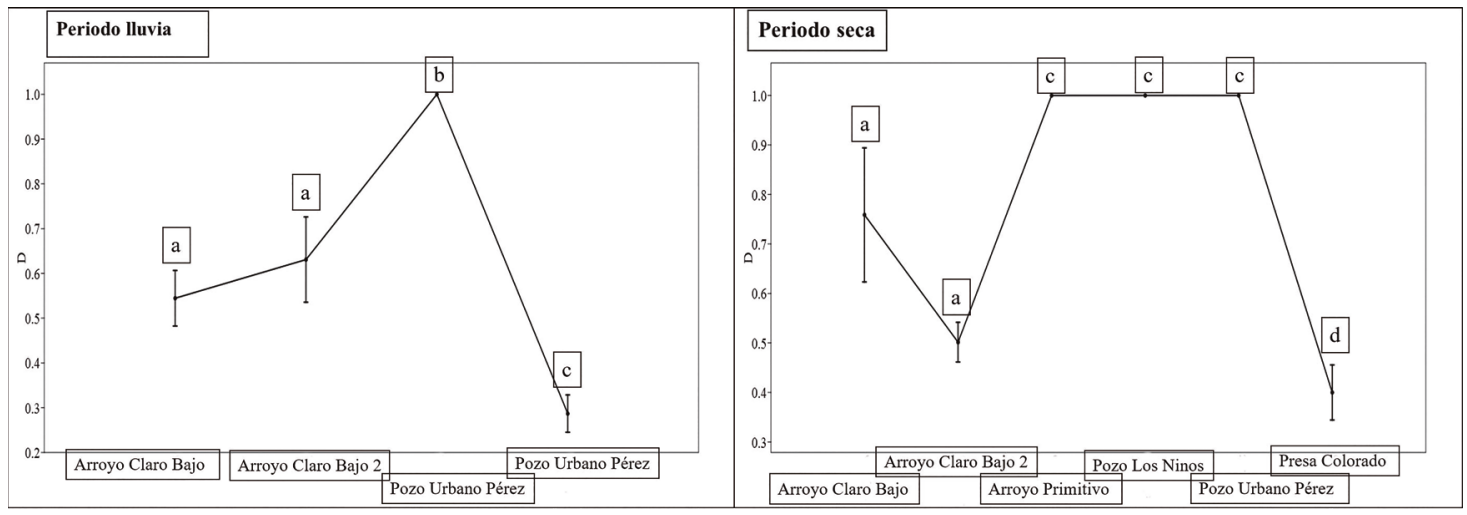

Figura 5. Dominancia (D) de especies entre sitios en diferentes épocas del año. Letras diferentes entre grupos significan diferencias estadísticas a partir de la prueba Permutación de Diversidad para 999 remplazamientos.

Presa Colorado fue el sitio de mayor diversidad ictiológica en el periodo poco lluvioso (1.17). Seguido de arroyo Clara Bajo 2 (0.76). La diversidad fue extremadamente baja en pozos los Ninos, Urbano Pérez y arroyo Primitivo. En el periodo lluvioso coincidió presa Colorado como el sitio de mayor diversidad (1.44), seguido de arroyo Claro Bajo (0.64) y arroyo Claro Bajo 2 (0.55), (Fig. 6).

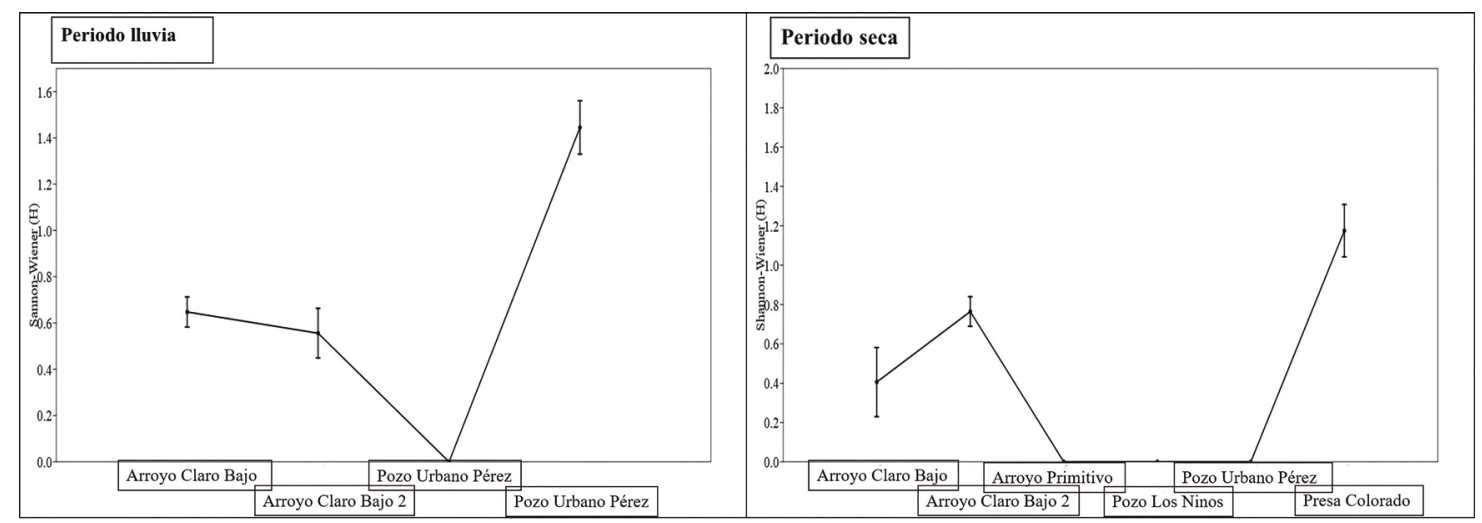

Figura 6. Diversidad de Shannon $(\mathrm{H})$ de especies entre sitios en diferentes épocas del año.

Diversidad Beta. Se obtuvo un valor de remplazamiento entre las comunidades de peces dulceacuícolas de 2.6 para la época de seca. Las comunidades ictiológicas que no presentaron intercambio ictiológico fueron pozo Los Ninos con presa Colorado; arroyo Primitivo y pozo Urbano Pérez (1.0) respectivamente entre cada par de sitios. Presa Colorado también presentó bajos índices de remplazamiento con el resto de los sitios, destacando con arroyo Claro Bajo 2 (0.80) y arroyo Claro Bajo (0.77). Encontrando los mayores remplazamientos de especies entre los arroyos Claro Bajo y Claro Bajo 2 (0.2). Además de arroyo Claro Bajo con arroyo Primitivo, pozos los Ninos y Urbano Pérez con un valor de 0.33 respectivamente para cada sitio (Tabla II). 
En la época de lluvia se obtuvo un índice de remplazamiento de 1.55 entre las comunidades ictiológicas, encontrando los más bajos remplazamientos entre presa Colorado con los arroyos Claro Bajo y Claro Bajo 2, valores de 0.75 respectivamente entre cada sitio. Las comunidades que compartieron mayor número de especies en esta época del año fueron arroyo Claro Bajo y arroyo Claro Bajo 2 con pozo Urbano Pérez (0.33), respectivamente para cada par de sitios (Tabla III).

Tabla II. Diversidad Beta de las comunidades ictiológicas en época de seca

\begin{tabular}{lcccccc}
\hline Localidades & $\begin{array}{c}\text { Arroyo } \\
\text { Claro Bajo }\end{array}$ & $\begin{array}{c}\text { Arroyo } \\
\text { Claro Bajo2 }\end{array}$ & $\begin{array}{c}\text { Arroyo } \\
\text { Primitivo }\end{array}$ & $\begin{array}{c}\text { Pozo los } \\
\text { Ninos }\end{array}$ & $\begin{array}{c}\text { Pozo Urbano } \\
\text { Pérez }\end{array}$ & $\begin{array}{c}\text { Presa } \\
\text { Colorado }\end{array}$ \\
\hline $\begin{array}{l}\text { Arroyo } \\
\text { Claro Bajo }\end{array}$ & 0 & 0.2 & 0.33 & 0.33 & 0.33 & 0.77 \\
$\begin{array}{l}\text { Arroyo } \\
\text { Claro Bajo 2 }\end{array}$ & 0.2 & 0 & 0.5 & 0.5 & 0.5 & 0.8 \\
$\begin{array}{l}\text { Arroyo } \\
\text { Primitivo }\end{array}$ & 0.33 & 0.5 & 0 & 1 & 0 & 0.75 \\
$\begin{array}{l}\text { Pozo los Ninos } \\
\text { Pozo Urbano }\end{array}$ & 0.33 & 0.5 & 1 & 0 & 1 & 1 \\
Pérez & 0.33 & 0.5 & 0 & 1 & 0 & 0.75 \\
Presa Colorado & 0.77 & 0.8 & 0.75 & 1 & 0.75 & 0 \\
\hline
\end{tabular}

Tabla III. Diversidad Beta de las comunidades ictiológicas en época de lluvia

\begin{tabular}{lcccc}
\hline Localidades & $\begin{array}{c}\text { Arroyo } \\
\text { Claro Bajo }\end{array}$ & $\begin{array}{c}\text { Arroyo } \\
\text { Claro Bajo2 }\end{array}$ & Pozo Urbano Pérez & $\begin{array}{c}\text { Presa } \\
\text { Colorado }\end{array}$ \\
\hline $\begin{array}{l}\text { Arroyo } \\
\text { Claro Bajo }\end{array}$ & 0 & 0 & 0.33 & 0.75 \\
$\begin{array}{l}\text { Arroyo } \\
\text { Claro Bajo 2 } \\
\begin{array}{l}\text { Pozo Urbano } \\
\text { Pérez }\end{array}\end{array}$ & 0 & 0 & 0.33 & 0.75 \\
Presa Colorado & 0.33 & 0.33 & 0 & 0.71 \\
\hline
\end{tabular}

Diversidad Gamma. En la época de seca se obtuvo una diversidad gamma igual a 7.37. La diversidad alfa comprendió el $34.01 \%$ y la diversidad beta el $65.98 \%$ de la diversidad gamma del paisaje. Para el periodo lluvioso se estableció una diversidad gamma de 6.89, comprendiendo la diversidad alfa el $39.91 \%$ y diversidad beta el $60.08 \%$ de las diversidades del conjunto de comunidades (Fig. 7). 


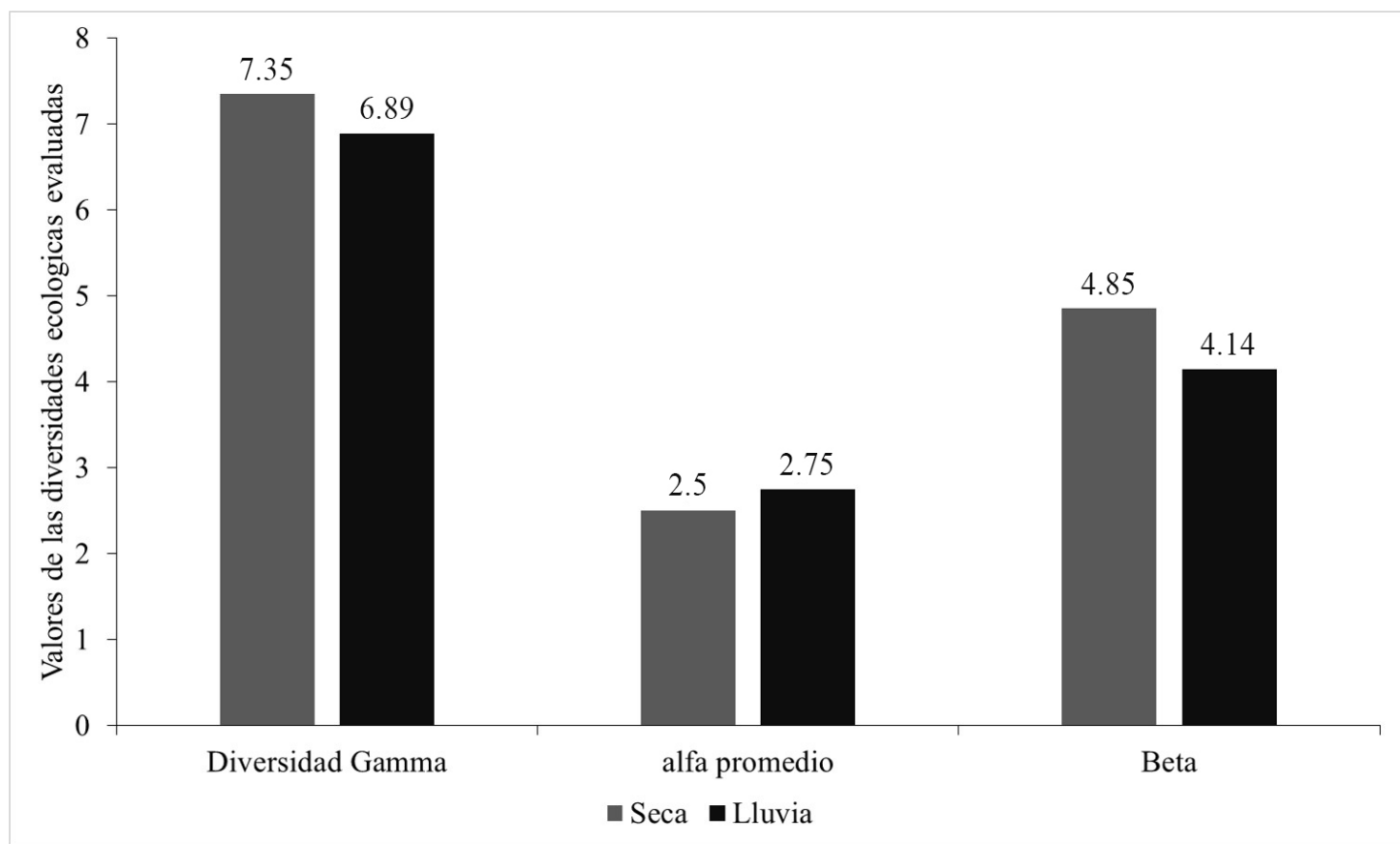

Figura 7. Diversidad gamma entre épocas del año.

Análisis de Similitud Ictiológica. Se obtuvieron tres grandes grupos en el periodo de seca a un $40 \%$ de similitud, para un $97 \%$ de correlación cofenética. El grupo I conformado por presa Colorado con un $100 \%$ de confiabilidad y presentando el más bajo porcentaje de similitud con pozo Los Ninos (0 \%) y arroyo Claro Bajo 2 (29\%). El grupo II constituido por la mayoría de los sitios para un $65 \%$ de robustez. Obteniéndose dentro de este grupo las mayores similitudes entre arroyo Primitivo con arroyo Claro Bajo $2(87.6 \%)$ y arroyo Claro Bajo 2 con pozo Los Ninos (79\%). El tercer grupo lo constituyó arroyo Claro Bajo 2, para un nivel de confiabilidad del $65 \%$ y $39 \%$ de similitud con los sitios del grupo II (Fig. 8).

Los tres grupos conformados en el periodo de seca en el análisis de similitud presentaron diferencias estadísticas con una fuerza de los factores sobre las muestras $\mathrm{R}=0.20 \mathrm{y}$ un nivel de significación $p=0.0131$. Siendo significativas entre grupo I con grupo II $(R=0.42$ y $p=0.0013)$ y grupo I con grupo III $(\mathrm{R}=0.37 \mathrm{y} \mathrm{p}=0.011)$. No se encontró diferencias entre grupo II con el grupo III (Fig. 8).

Para la época de lluvia se obtuvieron dos grandes grupos a un $45 \%$ de similitud, para un $97 \%$ correlación cofenética. El grupo I, conformado por arroyo Claro Bajo, para un $100 \%$ de robustez en la distribución de similitudes del dendrograma. Presentando arroyo Claro bajo la mayor similitud con arroyo Claro Bajo 2, 87.1 \%, y la menor similitud con presa Colorado $41.8 \%$. El grupo II está conformado por el resto de los sitios con un nivel de confiabilidad del $95 \%$. Se obtuvieron las mayores similitudes entre pozo Urbano Pérez y presa Colorado $84 \%$. Se obtuvo diferencias estadísticamente significativas entre los dos grupos estructurados en el dendrograma de similitud, en el periodo de lluvia con una fuerza de los factores sobre las muestras $\mathrm{R}=0.41 \mathrm{y}$ nivel de significación $\mathrm{p}=0.0022$ (Fig. 8). 
Las especies que más contribuyeron en la disimilitud entre los tres grupos formados en el dendrograma de similitud para la época de seca fueron: $G$. denticulatus $(30.39 \%)$, G. puncticulata $(13.61 \%)$ y G. punctata $(11.46 \%)$. A diferencia de L. macrochirus $(0.28 \%)$ y C. idella $(0.14 \%)$ como las especies que menos contribuyeron a la disimilitud (Tabla IV). Para el periodo de lluvia, las especies que más contribuyen en la disimilitud son: G. puncticulata $(13.71 \%)$, G. punctata $(9.38 \%$ ) y G. denticulatus $(6.22 \%)$. Presentando una menor contribución en esta época del año la especie L. macrochirus $(0.85 \%)$, (Tabla V).

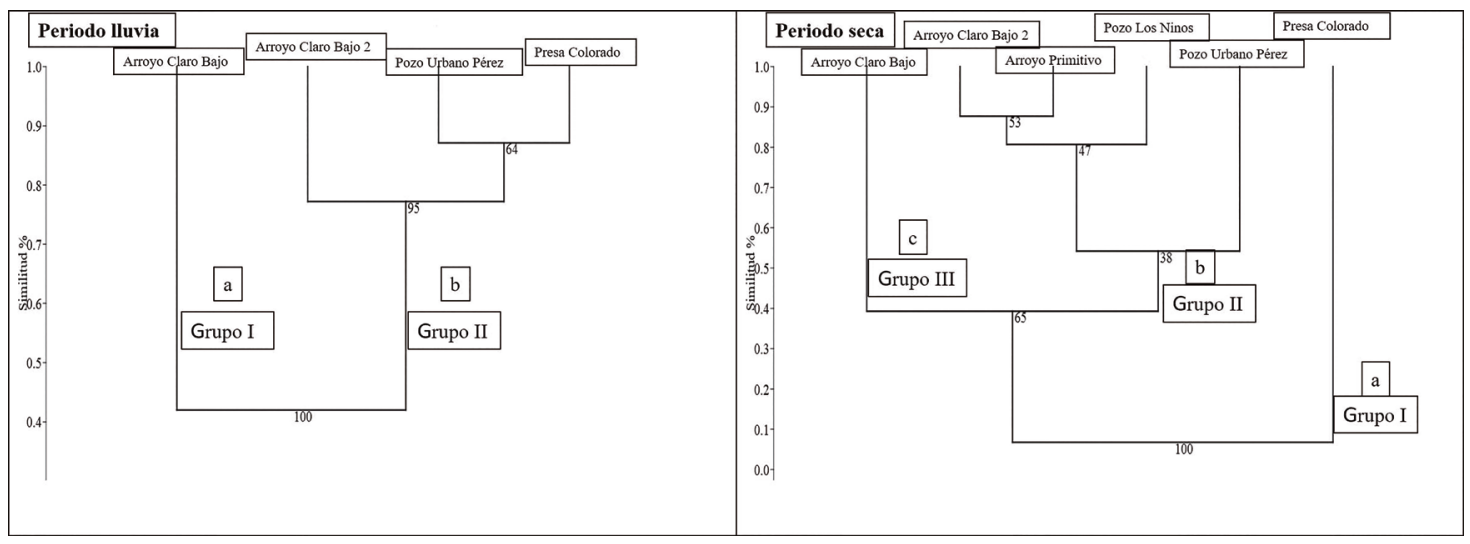

Figura 8. Dendrograma de similitud (\%) de los sitios de muestreo para cada época del año, a partir de la abundancia de las especies de peces. Correlación cofenética $=0.97$ en ambos periodos. Letras diferentes entre grupos significan diferencias estadísticas a partir de la prueba ANOSIM. Época de lluvia la fuerza de los factores sobre las muestras, $\mathrm{R}=0.41$ y nivel de significación $p=0.0022$. Época de seca la fuerza de los factores sobre las muestras, $\mathrm{R}=0.20 \mathrm{y}$ nivel de significación $p=0.0131$.

Tabla IV. Especies que más contribuyeron en la disimilitud entre los grupos formados en el análisis de similitud por el índice de Bray-Curtis en el periodo de seca. Promedio de disimilitud general 59.83

\begin{tabular}{lccc}
\hline \multicolumn{1}{c}{ Especies } & Promedio de disimilitud & Contribución (\%) & Acumulación (\%) \\
\hline Girardinus denticulatus & 30.39 & 50.8 & 50.8 \\
Gambusia puncticulata & 13.61 & 22.74 & 73.54 \\
Gambusia punctata & 11.46 & 19.16 & 92.7 \\
Guavina guavina & 1.82 & 3.04 & 95.75 \\
Gobiomorus dormitor & 0.98 & 1.64 & 97.39 \\
Nandopsis tetracanthus & 0.57 & 0.96 & 98.36 \\
Oreochromis aureus & 0.56 & 0.93 & 99.3 \\
Lepomis macrochirus & 0.28 & 0.46 & 99.77 \\
Ctenopharyngodon idella & 0.14 & 0.23 & 100 \\
\hline
\end{tabular}


Tabla V. Especies que más contribuyeron en la disimilitud entre los grupos formados en el análisis de similitud por el índice de Bray-Curtis en el periodo de lluvia. Promedio de disimilitud general 38.75

\begin{tabular}{lccc}
\hline Especies & Promedio de disimilitud & Contribución (\%) & Acumulación (\%) \\
\hline Gambusia puncticulata & 13.71 & 35.38 & 35.38 \\
Gambusia punctata & 9.38 & 24.23 & 59.61 \\
Girardinus denticulatus & 6.22 & 16.07 & 75.68 \\
Gobiomorus dormitor & 3.64 & 9.39 & 85.07 \\
Oreochromis aureus & 2.78 & 7.18 & 92.26 \\
Nandopsis tetracanthus & 2.14 & 5.52 & 97.79 \\
Lepomis macrochirus & 0.85 & 2.21 & 100 \\
\hline
\end{tabular}

\section{DISCUSIÓN}

Densidades en diferentes épocas del año. El total especies encontradas en el presente estudio representan el $15 \%$ de las 57 reportadas para Cuba (Ponce de León et al., 2014). En relación a la provincia de Holguín constituyen un $36 \%$ de las 25 especies reportadas (Vega et al., 2017). El endemismo ictiológico en Resbaladero es extremadamente bajo. Al ser reportadas solamente tres especies endémicas, estas representan un $13 \%$ de las 23 encontradas en Cuba y un 33 \% para las nueve especies registradas en Holguín (Ponce de León et al., 2014; Vega et al., 2017). Es importante destacar la perdida de especies endémicas en esta región. Vega et al. (2017) reportaron la ausencia de G. metallicus de los arroyos de esta localidad, anteriormente abundante en todos ellos. Además de Limia vittata especie encontrada en el 2015 en presa Colorado (Vega et al., 2017).

La especie Gambusia punctata es endémica, con amplia distribución en todos los arroyos y reservorios de aguas en Resbaladero. En el presente trabajo se observó una disminución de sus densidades en época de seca $\left(2.47 \mathrm{ind} / \mathrm{m}^{2}\right)$ y lluvia $\left(1.34 \mathrm{ind} / \mathrm{m}^{2}\right)$, encontrándose solamente en pozo Los Ninos, arroyo Claro Bajo y arroyo Claro Bajo 2.

Se encontró un total de tres especies introducidas, representando un $15 \%$ del total registradas para Cuba (Ponce de León et al., 2014). Encontrando un nuevo registro de especie introducida, Lepomis macrochrirus (pez sol) en presa Colorado. Es importante resaltar que no se logró la captura de Claria gariepinus en los sitios estudiados. Especie de amplia distribución en Cuba (Ponce de León et al., 2014), la misma capturada en elevadas cantidades por pescadores en presa Colorado, no así a partir del año 2019, producto a la muerte masiva de las mismas por causas desconocidas (Reynaldo y Hernández, 2019).

La especie Girardinus denticulatus es la que presenta las mayores densidades en ambos periodos del año y la de más amplia distribución. En el periodo de seca estuvo presente en cinco sitios y en lluvia en cuatro puntos de muestreo. Este resultado indica que esta especie cuenta con las mejores estrategias de adaptación en el área de estudio. Esta especie es endémica de Cuba, con distribución restringida solo a los macizos montañosos del centro y de Oriente. Se encuentra en zonas poco profundas de arroyos y ríos limpios, donde la corriente no es muy fuerte. 
Forma grupos grandes y se alimenta preferiblemente de algas y detrito, incluyendo invertebrados. Generalmente, convive con G. punctata (Ponce de León y Rodríguez, 2010). Ambas especies fueron detectadas conviviendo juntas en arroyo Claro y arroyo Claro Bajo 2 (Reynaldo y Hernández, 2019).

La especie Gambusia punticulata es la segunda con mayores densidades en ambas épocas del año y con una distribución restringida, registrada únicamente en presa Colorado. Es una especie antillana muy abundante en la mayoría de los cuerpos de agua cubanos, capaz de tolerar elevadas concentraciones de sales y contaminación. Se alimenta, principalmente, de insectos y larvas (Fong et al., 1997), aunque también incluye material vegetal en su dieta (Poey, 1854). Es muy abundante en estuarios y zonas cercanas al mar, además en zanjas albañales y en ríos eutrofizados (Ponce de León y Rodríguez, 2010).

Las especies que presentaron las densidades más bajas fueron: $C$. idella $\left(0.3 \mathrm{ind} / \mathrm{m}^{2}\right)$ en época de seca, no registrada en el periodo de lluvia, y $O$. aureus, con densidad de 0.83 y $1.24 \mathrm{ind} / \mathrm{m}^{2}$ en periodos de seca y lluvia, respectivamente. Ambas especies fueron detectadas únicamente en presa Colorado y constituyen especies exóticas invasoras. Las especies exóticas son aquellas que el hombre ha llevado fuera de su área natural de distribución, por razones económicas o de cualquier otra índole. Generalmente, tienen estrategias ecológicas diferentes a las especies autóctonas, lo que determina su éxito o fracaso adaptativo en diferentes ecosistemas. Interactúan con las especies nativas como depredadores o al establecer relaciones de competencia por los recursos del hábitat, ya sean alimentarios o de otro tipo. Muchas presentan determinadas adaptaciones reproductoras que limitan el éxito de las especies nativas. Algunas pueden ser dañinas, convirtiéndose en verdaderas plagas (Ponce de León y Rodríguez, 2010).

La reducida distribución de ambas especies exóticas en el área de estudio se debe a que constituyen especies que habitan en reservorios de aguas de elevada profundidad y sitios con mayor disponibilidad de alimento. El único lugar que cumple con ambos requisitos es presa de Colorado. Las bajas densidades de las especies en presa Colorado es producto a la pesca intensiva. Margalef (1995) y De Grado (1997) señalan que la práctica de pesca hace disminuir la densidad y diversidad específica de una comunidad dada.

La especie Oreochromis aureus es de origen africano que ha sido introducida en diferentes partes del mundo, principalmente en el trópico (Lovshin y Popma, 1995). Las especies exóticas en Cuba se han introducido para su cultivo en embalses, sin embargo, en muchos lugares han escapado de los centros de acuicultura, prosperando en ríos y presas, donde compiten por los recursos con muchas especies autóctonas, dada su resistencia, voracidad y elevada tasa de crecimiento (Rodríguez et al., 1993). En el caso de L. macrochirus (Pez sol), constituyen un nuevo registro para la provincia de Holguín. Es un pez introducido desde E.U.A, especie carnívora que altera la distribución y abundancia de los peces nativos, al igual que C. idella, de origen asiático (Reynaldo et al., 2016).

Análisis de los índices ecológicos. Conocer la diversidad biológica es uno de los objetivos más importantes de la biología (Serrato et al., 2013). Presa Colorado fue el sitio con la mayor riqueza de especies de peces en ambas épocas del año. Registrando dentro de este lugar todas las especies exóticas invasoras de la región, dos especies en cada periodo respectivamente $O$. aureus, C. idella y L. macrochirus. Estas especies presentan adaptaciones reproductoras y de alimentación superior a las autóctonas de la región (Ponce de León y Rodríguez, 2010). 
Los sitios que presentaron la más baja riqueza de especies fueron pozo los Ninos y arroyo Primitivo, con una sola especie en la época de seca y ninguna especie en la de lluvia. La pérdida de especies en la época de lluvia es producto del periodo prolongado de seca. Esto es acentuado por la modificación estructural del hombre en ambos sitios: remoción de los sedimentos del fondo para la mayor retención de agua. Las especies ausentes en ambos sitios son G. punctata en los Ninos y en arroyo Primitivo G. denticulatus.

Pozo Urbano Pérez presentó una sola especie en ambas épocas del año G. denticulatus. Especies endémicas de Cuba y de la región Oriental. Las sequias extremas constituyen el principal factor en reducir el número de especies de peces (Dahl, 1960). Sumado a este factor la acción del hombre, específicamente en esta región la construcción de la presa Colorado, la misma bloquea y limita el paso del agua hacia los arroyos y pequeñas escorrentías presentes en Resbaladero.

Las diferencias estadísticas en relación con la riqueza de especies están fuertemente relacionadas al tipo de ecosistema donde se encuentran. La menor riqueza fue encontrada en pozos artesanales, los mismos limitan la movilidad de los peces, además de reforzar la competencia interespecífica, desplazando la especie mejor adaptada al resto de las especies. Esta pudiera ser una de las razones de encontrar solamente una especie por cada pozo muestreado. El resto de los sitios fueron arroyos de pequeño caudal e intermitentes.

En los últimos decenios, la acrecentada influencia de la sequía ha dado lugar a que se le considere como uno de los mayores desastres naturales del mundo, el más frecuente y persistente, de mayores efectos negativos y con impactos adversos reales sobre el medio ambiente. Para Cuba, el impacto negativo de los persistentes y significativos eventos de sequía de corto y largo período acaecidos durante las últimas décadas han afectado significativamente la riqueza de especies dulceacuícolas (Ponvert-Delisles, 2016).

Las mayores equitatividades fueron encontradas en arroyo Claro Bajo 2 y Arroyo Claro Bajo, debido a las abundancias similares de G. punctata, G. denticulatus y N. tetracanthus, especies endémicas de Cuba, las dos últimas con distribución restringida. Es importante destacar que estas especies, a pesar de no estar en alguna categoría de amenaza, sí presentan altas probabilidades de desaparecer de Resbaladero, producto a las sequias extremas y a la acción negativa del hombre sobre sus ecosistemas (Reynaldo y Hernández, 2019).

La equitatividad en comunidades ictiológicas es altamente variable, un estudio realizado mensualmente en tres estaciones, fluctuaron entre 0.00 y 0.85 . Este es un comportamiento similar al de la diversidad, es decir, donde se presentaron los valores más altos de diversidad también se presentaron los altos valores de equitatividad y viceversa. Esto manifiesta la igualdad o desigualdad en la distribución de los individuos en la comunidad (Krebs, 1985). En el presente estudio se obtuvieron valores de equidad en el periodo de seca entre 0.00 y 0.69 y lluvia 0.00 y 0.93 . Donde se demuestra que en el periodo de lluvia existió una mayor disponibilidad de alimento y áreas a ocupar, lo que permite reducir la competencia entre especies y aumentar la equidad. El clima juega un papel importante en los procesos de dispersión y factores como la cantidad de precipitación y la reproducción, alimentación, crecimiento y temperatura constituyen los principales componentes que enmarcan los ecosistemas dulceacuícolas tropicales (Ramírez y Viña, 1998). 
Los peces de agua dulce se adaptan mejor en aguas eutróficas y profundas (Ramírez y Viña, 1998) y sitios con variedad de hábitats, donde se presenta mayor diversidad de especies y menor grado de dominancia (Barrela et al., 1994). Es importante destacar que no coincidieron los mismos sitios de mayor equidad con el de mayor diversidad en el presente trabajo. Para este estudio, arroyo Claro Bajo 2 y arroyo Claro Bajo fueron los sitios con mayor equidad ictiológica en ambas épocas del año y a su vez no fueron los de mayor diversidad.

No se encontró equitatividad en los pozos Primitivo, Urbano Pérez y los Ninos, debido a la presencia de una sola especie, este resultado sugiere que estos sitios en el período de seca no son capaces de sostener más de una especie de peces. Las alteraciones de los paisajes por la acción del hombre, trae consecuencias negativas a múltiples escalas, modificando la dinámica ecológica en los sistemas de agua dulce (Wiens et al., 2006). Resbaladero es un área altamente deforestada de forma ilícita, principalmente la vegetación riparia. La vegetación riparia es una fuente primordial de materia orgánica para los arroyos, pequeños reservorios y la ictiofanuna (Lourenço et al., 2021).

La mayor dominancia fue obtenida en arroyo Primitivo y pozos los Ninos y Urbano Pérez, sitios que presentaron una sola especie en ambos periodos del año caso de pozo Urbano Pérez. A diferencia de arroyo Primitivo y pozo los Ninos donde en el mes de lluvia no se encontró ninguna especie ictiológica. Producto a la extrema sequía que se manifestó en el área estudio. Es importante resaltar que las especies registradas en los sitios antes mencionadas son endémicas de Cuba (Ponce de León y Rodríguez, 2010).

Los sitios con las mayores dominancias constituyen reservorios de agua de poco caudal e intermitentes, llegando a quedarse sin agua cuando la ausencia de precipitaciones es extrema. Margalef (1995) menciona que, si existe una especie dominante en número sobre otras en una comunidad, las diversidades son bajas. Existe una relación inversa entre la diversidad específica y la dominancia numérica, resultados similares fueron reportados por Méndez et al. (2004), quienes señalan que la presencia de especies dominantes resulta en comunidades de baja diversidad.

Los sitios que presentaron los mayores valores de Dominancia fueron los mismos que presentaron la menor riqueza de especie, Pozo Primitivo, Urbano Pérez y los Ninos, ambos con valor de uno. Una característica ecológica posible es el desplazamiento entre especies por los recursos del ecosistema, estableciéndose una sola especie como la dominante con una elevada abundancia (Ponce de León et al., 2014).

Presa Colorado fue el sitio con la menor dominancia registrada en ambos periodos, presentando diferencias estadísticas con el resto de los sitios. El $50 \%$ de las especies de peces registradas en este lugar son exóticas e invasoras. Las mismas pudieran estar desplazando al resto de las especies ictiológicas endémicas de la región hacia sitios con menos recursos de alimento, refugio, movilidad y espacio por individuo, además de las interacciones bióticas entre diferentes especies (Reynaldo et al., 2021).

Las bajas diversidades encontradas en este estudio podrían estar relacionadas con la dominancia numérica, ya que en ambas épocas se presentó una dominancia de diferentes especies en $100 \%$ sobre las otras, obteniéndose las diversidades más bajas para las estaciones. La ictiofauna en su conjunto se acomoda mejor en aguas eutróficas, donde su diversidad y abundancia aumenta (Torres et al., 2006). 
La diversidad ictiológica presenta una estrecha relación con el funcionamiento de los ecosistemas, a través de procesos como la productividad y la estabilidad, y por su modificación como resultado de las actividades humanas (Maclaurin y Sterelny, 2008).

Es importante destacar que a pesar de que presa Colorado tiene una mayor diversidad no constituye una prioridad para la conservación, debido a que este sitio presenta abundantes especies exóticas. A diferencia de arroyo Claro Bajo 2, sitio importante para la conservación, por presentar un mayor número de especies endémicas con abundancias equitativas y mayor diversidad (Moreno et al., 2011).

Presa Colorado presentó una diversidad superior en $117 \%$ y $144 \%$ en ambas épocas del año a las obtenidas en arroyo Primitivo y pozos Urbano Pérez y Los Ninos. El mayor contraste de la diversidad entre épocas se detectó en el sitio arroyo Claro Bajo 2, en el cual en la época de sequía la diversidad fue superior en 1.38 veces a la diversidad obtenida en lluvia. Otro sitio con marcada diferencia fue presa Colorado, con una diversidad 0.81 superior en la época de lluvia a la registrada en seca.

Estos resultados permiten inferir que arroyo Claro Bajo 2, constituye un sitio de reclutamiento de especies ictiológicas durante la época de seca extrema. Lugar donde el $100 \%$ de las especies registradas fueron endémicas en ambos periodos. Por esta razón, es de notable importancia la conservación de este lugar para mantener la diversidad ictiológica en Resbaladero. Las diferencias entre las diversidades permiten explorar la influencia de los procesos ecológicos y evolutivos en la abundancia de las especies coexistentes. Procesos ecológicos como la tasa de nacimiento, mortalidad, inmigración y emigración intervienen en la abundancia de las especies y actúan bajo la influencia de distintos factores bióticos y abióticos (Begon et al., 2006).

Diversidad Beta. La diversidad beta o diversidad entre hábitats es el grado de reemplazamiento de especies o cambio biótico a través de gradientes ambientales (Whittaker, 1972). El valor obtenido en la época de seca fue 1.71 veces superior al obtenido en la época de lluvia. Este resultado nos permite inferir que en el periodo de seca existieron menos recursos de alimento, refugio, movilidad de los peces y espacio por individuo. Propiciando mayores interacciones bióticas entre diferentes especies, dando como resultado mayor remplazamiento (Reynaldo et al., 2021). Los sitios con los mayores intercambios en época de seca fueron: arroyo Claro Bajo con arroyo Claro Bajo 2; además de arroyo Claro Bajo con arroyo Primitivo. Estos sitios mantuvieron una pequeña conectividad del agua en la época de pocas precipitaciones. A diferencia de pozo Los Ninos con presa Colorado, arroyo Primitivo y pozo Urbano que no existió intercambio de especies.

En la época de lluvia coincidió presa Colorado como el sitio que menos especies compartió, principalmente con arroyos Claro Bajo y Claro Bajo 2, producto de la barrera de contención del agua de la presa que limita la movilidad de las especies de peces entre un lugar a otro. A diferencia de pozo Urbano Pérez, que compartió la especie G. denticulatus con los arroyos antes mencionados. Estos sitios presentan una comunicación entre ellos cuando ocurren fuerte precipitaciones. Las especies más compartidas entre sitios para ambas épocas del año son: $G$. punctata y $G$. denticulatus. Indicando que ambas especies presentan como estrategia ecológica el remplazamiento entre diferentes estaciones, este comportamiento coincide con el reportado por Ponce de León y Rodríguez (2010). 
Diversidad Gamma. La diversidad gamma constituye una aplicación poderosa para analizar la participación de sus componentes de medida de las diversidades alfa y beta. Si la medición de la diversidad alfa ha sido sumamente compleja, la base conceptual y metodológica del componente beta lo es aún más (Tuomisto, 2010). Las diversidades gammas registradas en el área de estudio tuvieron un mayor aporte de la diversidad beta en ambos periodos del año. Siendo 1.09 veces mayor en seca con respecto al periodo lluvioso. Este resultado indica que las comunidades ictiológicas presentes en Resbaladero están ecológicamente estructuradas por el grado de diferenciación entre las especies o el grado de remplazamiento entre especies a través del gradiente ambiental (Moreno, 2001).

Jost (2010) estableció que la estimación de la diversidad gamma en una región constituye el aporte teórico más importante en la medición de la diversidad desde que Whittaker introdujo el concepto de la diversidad beta (Ellison, 2010). La diversidad gamma establece la diversidad regional, y de ella se puede derivar análisis que puedan ayudar a la conservación efectiva de dicha diversidad (Jost et al., 2010).

Análisis de Similitud Ictiológica. En la época de seca se obtuvieron tres grandes grupos, constituido el grupo II con el mayor número de sitios y a su vez con las mayores similitudes ictiológicas. Adiferencia del grupo I y III, constituidos por un solo grupo cada uno y con menores similitudes. Las especies que más contribuyeron en la disimilitud fueron: $G$. denticulatus, G. punticulata y G. punctata, especies de la familia Poeciliidae. Girardinus denticulatus ha sido mencionada anteriormente como una especie que contribuyó en un mayor porcentaje de disimilitud en las comunidades ictiológicas de los ríos del municipio Gibara (Reynaldo et al., 2021). Gambusia puncticulata y G. punctata constituyen especies con una distribución amplia en el municipio de Gibara (Reynaldo et al., 2016). Además, son especies muy bien establecidas en Cuba (Ponce de León, 2011).

Las diferencias estadísticas encontradas entre los grupos del dendrograma en época de seca pueden estar determinados por las características físicas y ecológicas de cada sitio. Grupo I constituido por presa Colorado, sitio artificial y con un elevado volumen de agua; grupo III formado por arroyo Claro Bajo, único lugar con agua en ambos periodos del año con un volumen de agua intermedio, y el grupo II conformado por el resto de los sitios que presentan una limitada capacidad de retención de agua y son intermitentes en el periodo de seca.

En el periodo de lluvia se obtuvieron dos grandes grupos. El primero formado por arroyo Claro Bajo y grupo II por el resto de los sitios estudiados en esa época del año. Presentando este último las mayores similitudes ictiológicas. Las especies que más influyeron en la disimilitud fueron las mismas especies registradas en la época de seca. Las diferencias estadísticas entre ambos grupos pueden ser inferidas de las características antes mencionadas en la época de seca.

\section{CONCLUSIÓN}

Se registró un total de nueve especies ictiológicas en Resbaladero, presentando presa Colorado el valor más alto de riqueza y de diversidad. Arroyo Claro Bajo presentó la mayor equitatividad en la época de seca y arroyo Claro Bajo 2 en la época de lluvia. Se obtuvieron tres grupos de similitud en el periodo de seca y dos en lluvia, contribuyendo en un porcentaje mayor de disimilitud las especies $G$. denticulatus, $G$. punticulata y $G$. punctata en ambos periodos. 


\section{AGRADECIMIENTOS}

Este estudio se realizó gracias al aporte del proyecto de investigación (Disminución de la vulnerabilidad de una comunidad rural y sus ecosistemas ante la variabilidad climática en la localidad de Resbaladero, Rafael Freyre, Holguín, Cuba. Etapa II). Este proyecto pertenece al Centro de Investigaciones y Servicios Ambientales de Holguín.

\section{LITERATURA CITADA}

Barrela, W., C. Beaumord y M. Petrere. 1994. Comparación de la comunidad de peces de los ríos Manso (MT) y Jacaré Pepira (Sp), Brasil. Acta Biológica Venezolana, 15 (2): 11-20.

Begon, M., C. R. Townsend y J. L. Harper. 2006. Ecology: from individuals to ecosystems, Cuarta edición. Blackwell, Oxford, 738 pp.

Clarke, K. R. 1993. Non-parametric multivariate analysis of changes in community structure. Australian Journal of Ecology, 18: 117-143.

Dahl, G. 1960. Los peces de los ríos colombianos. El estudio y la defensa de una gran riqueza natural, Bogotá. Revista Nacional de Agricultura, 1: 22-24.

De Grado, A. 1997. Estudio de la ictiofauna de Ensenada Grande del Obispo (Laguna Grande), Estado Sucre, Venezuela. Trabajo de Grado de Maestría en Biología Pesquera, Universidad de Oriente, Cumaná, Venezuela, 45 pp.

Ellison, A. M. 2010. Partitioning diversity. Ecology, 91: 1962-1963.

Fong, A. G., G. G. Garcés y E. F. Portuondo. 1997. Invertebrados en la alimentación de Gambusia punctata (Cyprinodontiformes: Poeciliidae) en aguas marinas. Cocuyo, 5: 13-14.

Hammer, Ø., D. A. T. Harper y P. D. Ryan. 2001. PAST: Paleontological Statistics Software Package for Education and Data Analysis. Palaeontologia Electronica, 4: 1-9.

Harper, D. A. T. (eds.). 1999. Numerical Palaeobiology. John Wiley \& Sons, New York, EEUU, $233 \mathrm{pp}$.

Jost, L. 2010. The relation between evenness and diversity. Diversity, 2: 207-232.

Jost, L., P. DeVries, T. Walla, H. Greeney, A. Chao y C. Ricotta. 2010. Partitioning diversity for conservation analyses. Diversity and Distribution, 16: 65-76.

Krebs, C. 1985. Ecología: Estudio de la distribución y abundancia. 2da. Ed. Harla, S.A. de C.V. México, $753 \mathrm{pp}$.

Landen, R. 1996. Statistics and partitioning of species diversity, and similarity among multiple communities. Oikos, 76 (1): 5-13.

Lourenço, B. G., L. C. Gontijo y P. Gerhard. 2021. A ecologia de peixes de riacho sob a perspectiva da ecologia de paisagens. Oecologia Australis, 25 (2): 475-493. https://doi.org/10.4257/oeco.2021.2502.16 
Lovshin, L. L. y T. J. Popma. 1995. Commercial production of tilapia fry and figerlings. Situación del cultivo de la tilapia en Panamá. Memorias del I Simposium Centroamericano sobre cultivo de tilapia, Eds. Pradepesca, Incopesca, Acuacorporacion y Universidad Nacional Heredia Costa Rica, 5: 95-101.

Maclaurin, J. y K. Sterelny. 2008. What is biodiversity? The University of Chicago Press, Chicago, $244 \mathrm{pp}$.

Magurran, A. E. 1988. Ecological diversity and its measurement. Princeton University Press, New Jersey, 179 pp.

Margalef, R. 1995. Ecología. Eds. Omega, S.A. Barcelona-España, 951 pp.

Méndez, E., J. L. Ruiz, J. A. Torres, Á. M. Eugenía, A. Fariña, S. Sant, A. Prieto, J. Barrios y B. Marin. 2004. Nuevos registros para la ictiofauna marina del parque nacional Mochima, Venezuela. Boletín del Centro de Investigaciones Biológicas, 38: 21-31.

Moreno, E. C., F. Barragán, E. Pineda y P. P. Pavón. 2011. Reanálisis de la diversidad alfa: alternativas para interpretar comparar información sobre comunidades ecológicas. Revista Méxicana de Biodiversidad, 82: 1249-1261.

Moreno, C. E. 2001. Métodos para medir la biodiversidad. M\&T-Manuales y Tesis SEA, vol. 1. Zaragoza, España, 84 pp.

Poey, F. 1854. Los guajacones, pececillos de agua dulce. Memorias de la Historia Natural de la Isla de Cuba, 1 (32): 374-390.

Ponce de León, J. L. 2011. La ictiología de aguas dulces. Línea de investigación en la Facultad de Biología de la Universidad de la Habana. Boletín de la Sociedad Cubana de Zoología, 1: 19-25.

Ponce de León, J. L., C. M. Acosta, A. M. Uribe, y M. E. García, 2014. Biología de Peces dulceacuícolas de Cuba. Revista Anales de la Academia de Ciencias de Cuba, 4 (2): 1-10.

Ponce de León, J. L. y R. S. Rodríguez. 2010. Peces cubanos de familia Poeciliidae. Guía de campo. Editorial Academia, La Habana, 38 pp.

Ponvert-Delisles, B. D. R. 2016. Algunas consideraciones sobre el comportamiento de la sequía agrícola en la agricultura de Cuba y el uso de imágenes por satélites en su evaluación. Revista de Cultivos Tropicales, 37 (3): 22-41.

Quammen, D. 1996. The song of the Dodo: island biogeography in an age of extinction, Scriber, $702 \mathrm{pp}$.

Ramírez, A. y G. Viña. 1998. Limnología Colombiana. Aportes a su conocimiento y estadísticas de análisis. Colombia. Bogotá. Editorial Panamericana, 292 pp.

Reynaldo, C. E. y M. D. Hernández. 2019. Informe parcial del estudio de las comunidades de peces, reptiles y anfibios, del proyecto: Disminución de la vulnerabilidad de una comunidad rural y sus ecosistemas ante la variabilidad climática en la localidad de Resbaladero, Rafael Freyre, Holguín, Cuba. CISAT, 63 pp. 
Reynaldo, C. E., M. C. Vega, T. A. Vega. y G. E. Córdova. 2021. Co-ocurrencia de peces fluviátiles en los ríos de Gibara, Holguín, Cuba. Novitates Caribaea, 17: 15-31.

Reynaldo, C. E., T. A. Vega, V. A. Fernández y G. E. Córdova. 2016. Distribución y similitud de los peces dulceacuícolas del municipio Gibara, Holguín, Cuba. Novitates Caribaea, 10: 71-86.

Rodríguez, H. G., G. S. Salazar y J. L. Polo. 1993. Estudio nacional de Colombia. En Diagnóstico sobre el Estado de la Acuicultura en América Latina y el Caribe, Síntesis regional GCP/RLA/102/ITA. Aquila II. FAO. Documento de Campo, 11: 162-165.

Serrato Díaz, A., A. C. Romero y O. A. Castro. 2013. La biodiversidad y evolución en ambientes acuáticos analizadas con herramientas moleculares. Hidrobiológica, 23 (3): 275-286.

Torres, Y., G. Roldán, S. Asprilla y T. S. Rivas. 2006. Estudio preliminar de algunos aspectos ambientales y ecológicos de las comunidades de peces y macroinvertebrados acuáticos en el río Tutunendo, Chocó, Colombia. Revista Académica Colombiana de Ciencia, 30 (114): 67-76.

Tuomisto, H. 2010. A diversity of beta diversities: straightening up a concept-gone awry. Part 1. Defining beta diversity as a function of alpha and gamma diversity. Ecography, 33: 2-22.

Vega, T. A., C. E. Reynaldo, V. A. Fernández, B. F. Ocaña y G. N. Guerra. 2017. Peces dulceacuícolas de Holguín. Poeyana, 504: 33-37.

Whittaker, R. H. 1972. Evolution and measurement of species diversity. Taxon, $21(2 / 3): 213-251$.

Wiens, J. J., C. H. Graham, D. S. Moen, S. A. Smith y T. W. Reeder. 2006. Evolutionary and ecological causes of the latitudinal diversity gradient in Hylid frogs: treefrog trees unearth the roots of high tropical diversity. The American Naturalist, 168: 579-596.

Cómo citar: Reynaldo-De la Cruz, E., Hernández-Marrero, D. F., Urbino-Rodríguez, J., \& Ayra-Cedeño, R. (2022). Diversidad de los peces dulceacuícolas en Resbaladero, Holguín, Cuba. Novitates Caribaea, (19), 43-61. https://doi.org/10.33800/nc.vi19.289. Artículo científico original. 